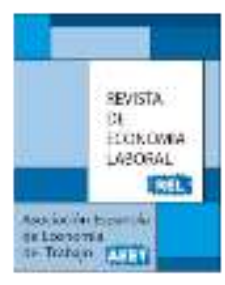

\title{
RECOMENDACIONES BIBLIOGRÁFICAS PARA EL ESTUDIO DE LA INFORMALIDAD LABORAL ${ }^{1}$
}

\author{
Jesuswaldo Martínez Soria \\ Universidad Nacional Autónoma de México
}

\section{Introducción}

La informalidad constituye un conjunto de prácticas productivas y laborales que, al reproducirse al margen de la legislación y propiciar múltiples afectaciones socioeconómicas, se convierte en un problema complejo que requiere de distintos esfuerzos académicos para mejorar su comprensión. La literatura especializada, que ha estudiado desde distintas perspectivas el problema de la informalidad, ha aportado múltiples evidencias sobre su comportamiento. De ese extenso acervo de estudios, se advierte que la actividad informal está relacionada con distintos desequilibrios en el mercado de trabajo — como la precariedad y la desprotección del empleo, los bajos salarios, la baja productividad, la segmentación y la exclusión laborales, entre otros-, y con anomalías en los mercados de bienes y servicios, que encuentran en las prácticas informales un riesgo para la competencia, la producción y la comercialización.

Asimismo, la informalidad afecta la gestión de las instituciones fiscales y de seguridad social, con consecuencias negativas en las fuentes de financiamiento y en los servicios públicos, y está involucrada en la evolución de la pobreza, la desigualdad del ingreso, el bienestar social y el desarrollo económico. Cabe destacar que con las prácticas de la informalidad se vulnera la satisfacción de los derechos sociales, reconocidos tanto en la normatividad internacional como en la nacional, cuya cobertura busca garantizar el acceso a la salud, a la seguridad social, al trabajo decente, así como el derecho al desarrollo.

\footnotetext{
${ }^{1}$ Las reflexiones y recomendaciones bibliográficas que aquí se presentan son retomadas del texto Informalidad: implicaciones legislativas y políticas públicas para reducirla, editado por el Instituto Belisario Domínguez del Senado de la República en México.
}

(C) Revista de Economía Laboral 
El conocimiento actual sobre el fenómeno de la informalidad, constituye un avance fundamental para mejorar el marco de actuación de la política pública y representa una plataforma para abrir líneas de investigación que aporten nuevas evidencias. En el transcurso de las últimas tres décadas, el conocimiento internacional sobre las causas, la evolución y las implicaciones de la actividad económica informal, ha logrado un desarrollo significativo a través de múltiples vertientes: fundamentación teórica, evidencia empírica, información estadística, metodologías y técnicas de estimación, marco legal y políticas públicas. Estos avances suponen también que el interés por comprender y solucionar las anomalías asociadas a la informalidad, se ha extendido entre los diversos actores sociales; incluyendo a quienes tienen la responsabilidad de formular y aplicar estrategias públicas y de vigilar el Estado de derecho.

Durante ese tiempo, se han logrado mejorar las fuentes de información estadística y los métodos de medición de la informalidad y se han establecido lineamientos internacionales para su definición (OIT, 2013). El registro histórico de las estadísticas sobre empleo informal da cuenta de un fenómeno estructural relacionado estrechamente con la evolución de los ciclos económicos. Ahora, se tienen más y mejores indicadores para conocer con mayor exactitud el desempeño y la composición de las actividades informales, lo que ofrece elementos que ayudan en el diseño, la instrumentación, el seguimiento y la evaluación de las políticas públicas.

La compleja configuración de la informalidad como un problema público representa un desafío significativo para el Estado. Por ello, resulta indispensable mejorar el diseño y la instrumentación de las políticas públicas, con base en el conocimiento ofrecido por la investigación académica y por las experiencias internacionales que permiten identificar las intervenciones públicas de mayor eficacia en la formalización.

Por estas razones, se ofrece en esta colaboración una serie de recomendaciones bibliográficas que constituyen un acervo básico en el estudio de la informalidad laboral. Las referencias bibliográficas están integradas en tres secciones. La primera incluye textos sobre la teoría y medición estadística. En la segunda se refieren estudios en los que se tratan los determinantes de la informalidad y su relación con diversas variables que intervienen en el desempeño económico, la ocupación, la pobreza y la desigualdad, entre otras. En la tercera sección se consideran textos en los que se aborda el autoempleo, teniendo en cuenta que esta 
modalidad es uno de los pilares más importantes de la informalidad laboral.

\section{Teoría y medición estadística.}

Los estudios que se esfuerzan por constituir un cuerpo teórico de la informalidad, incluyen propuestas conceptuales en torno al sector informal vinculadas a la economía laboral, la economía no regulada y a lo que en países desarrollados se ha denominado como economía sumergida. Se han realizado también fundamentos teóricos bajo el esquema conceptual que ha sido desarrollado por los organismos internacionales, en particular por la OIT mediante el Grupo Delhi. Asimismo se ha abordado la perspectiva conceptual del trabajo decente y su incorporación en la legislación.

A la par de los avances conceptuales, se incluyen directrices para la medición estadística del empleo informal, con fuentes de información y métodos de estimación cada vez mejores. En este cuerpo de estudios se construyen distintos indicadores de empleo informal y se utilizan métodos econométricos y estadísticos. Destaca la incorporación de modelos sectoriales para el estudio de los mercados de trabajo en los países en desarrollo y los análisis de las trayectorias laborales en distintas actividades, sectores y unidades de diferentes tamaños.

\section{Referencias}

Azuma, Y. y Grossman, H. (2002). "A theory of the informal sector". National Bureau of Economic Research, Working Paper 8823.

Blades, D. y Roberts, D. (2002). "Measuring the non-observed economy”. OECD Statistics Inbrief, 5 .

Blanchard, O. y Leigh, D. (2013). Growth Forecast Errors and Fiscal Multipliers. IMF Working Paper, 13/1.

Bovi, M. (2003). The Nature of the Underground Economy - Some Evidence from OECD Countries. IMAD JIIDT, 7.

Buehn, A. y Schneider, F. (2011). "Shadow economies around the world: novel insights, accepted knowledge, and new estimates". International Tax Public Finance, 19, 139-171. Cassel, D. (1983). The Growing Shadow Economy: the Implications for Stabilization Policy. Paper Prep. for the Conference on The Economics of the Shadow Economy, Bielefeld.

De Soto, H. (1986). “El otro sendero. La revolución informal.” Editorial El Barranco. 
Espinoza, M. (2003), Trabajo decente y protección social. OIT, Santiago de Chile. Fields, G. (2008). "Guía para los modelos multisectoriales de trabajo en los países en desarrollo". El Trimestre Económico, vol. LXXV (2), núm. 298, abril-junio, 2008, pp. 257297

Frey, B. S. y Pommerahane, W. W. (1984). "The Hidden Economy. State and Prospects for Measurement". Review of income and Wealth, 30(1).

Ghai, D. (2003), Trabajo decente. Concepto e indicadores. Revista internacional del trabajo, 122(2), 125-160.

Guergil, M. (1988). "Algunos alcances sobre la definición del sector informal." Revista de la CEPAL, No. 35.

Gyomai, G. y Ven, P. van de. (2014). The Non-Observed Economy in the System of National Accounts. OECD, Statistics Brief, 18.

Hart, K. (1972). "Employment, incomes and equality: A strategy for increasing productive employment in Kenya.” International Labour Office, Génova.

Henley, A., G. Reza y F. Carneiro, (2006). "On Defining and Measuring the Informal Sector.” Discussion Paper No. 2473, Institute for the Study of Labor (IZA).

Khamis, M. (2012). "A note on informality in the labour market". Journal of International Development, pp. 894-908.

Kholodilin, K. A. y Thiessen U. (2011). The Shadow Economy in OECD Countries: PanelData Evidence. Institute for Economic Research, Berlin, DIW Berlin Discussion Paper, 1122.

Loayza, N.V. (1996). "The Economics of the Informal Sector: A simple Model and Some Evidence from Latin America”. Carnegie-Rochester Conference Series on Public Policy, $45,129-62$.

Lubell, H. (1991). "The informal sector in the 1980's and 1990's." OECD, París.

Maloney, W. F. (2003a). "Informality revisited." Policy Research Working Paper 2965, The World Bank.

Negrete, R. (2011). "El concepto estadístico de informalidad y su integración bajo el esquema del Grupo de Delhi”. Realidad, datos y espacio, Revista Internacional de Estadística y Geografía, vol. 2 Núm. 3, septiembre-diciembre, pp. 77-94.

Organización para la Cooperación y el Desarrollo Económico (OECD). (2002). Measuring the Non Observed Economy: A Handbook. International Labour Office, International Monetary Fund, International Statistical Committee of the Commonwealth of Independent States, Paris: OECD Publishing. Recuperado de http://www.oecd.org/std/na/1963116.pdf. 
Organización Internacional del Trabajo (OIT). (2015). Trabajo decente. Memoria del Director General a la $87^{a}$ reunión de la Conferencia Internacional del Trabajo (Ginebra, OIT).

(2013). La medición de la informalidad: Manual estadístico sobre el sector informal y el empleo informal. Oficina Internacional del Trabajo, Ginebra.

(2003). Directrices sobre una definición estadística de empleo informal, adoptadas por la Decimoséptima Conferencia Internacional de Estadísticos del Trabajo. La Decimoséptima Conferencia Internacional de Estadísticos del Trabajo (CIET).

(2002). El trabajo decente y la economía informal. Conferencia

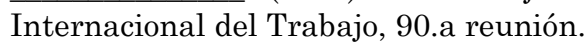

Portes, A. (2010). "Chapter Title: The Informal Economy". Princeton University Press, JSTOR, pp. 130-161.

Portes, A. (1995). En torno a la informalidad: ensayos sobre teoría y medición de la economía no regulada, FLACSO México.

Roubaud, F. (1995). "La economía informal en México.” Ed. Fondo de Cultura Económica.

Ruesga, S. (2000). "La economía sumergida”. Editorial Acento, Madrid.

(1986). "Economía oculta: de la definición y de los métodos de estimación". En

Luis Ruiz-Maya (Coord.). Problemas estadísticos de la economía sumergida. Madrid: Instituto Nacional de Estadística.

Schneider, F. y Buehn, A. (2016). "Estimating the Size of the Shadow Economy: Methods, Problems and Open Questions". IZA, Discussion Paper No. 9820.

Schneider, F. (2014) "The Shadow Economy and Shadow Labor Force: A Survey of Recent Developments". IZA, Discussion Paper No. 8278.

(2014). "Shadow Economies in 25 Latin American and Caribbean Countries: What Are the Driving Forces?" IZA, Department of Economics, Johannes Kepler University of Linz.

Tanzi, V. (1999). "Uses and abuses of Estimates of the Underground Economy". The Economic Journal, 109(456), 338-340.

Abroad. Lexington, MA.

(1982). (Ed.). The Underground Economy in the United States and

(1980). "The underground economy in the United States: Estimates and implications". Banca Nazionale del Lavoro, 135:4, pp. 427-453.

Thomas, J. J. (1992). "Informal economic activity.” LSE Handbooks in Economics. 


\section{Características, dinámica y determinantes de la informalidad.}

En este cuerpo de literatura se exploran las principales características y determinantes de la informalidad laboral. Los estudios desarrollados discuten y contrastan empíricamente la relación existente entre la informalidad y distintas variables relacionadas tanto con el desempeño económico, como con las características de los trabajadores.

Se incorporan estudios que abordan la relación entre la informalidad y el ciclo económico. Asimismo, se trata el tema de la fiscalidad como una de las principales determinantes de este fenómeno. Se analiza también el carácter voluntario o involuntario de la informalidad y el posible efecto de las leyes laborales en esta condición. Se estudia, por otra parte, el comportamiento del sector informal ante la liberalización comercial y la relación de este fenómeno con las instituciones públicas. Se profundiza de manera particular la relación entre la informalidad y la escolaridad y se propone que la informalidad laboral está ligada además a otras condiciones de alta vulnerabilidad del trabajador (baja escolaridad, alta marginación, mujeres con hijos pequeños, jóvenes y adultos mayores). Se aborda también el tema de la relación que tiene la informalidad con la pobreza y la desigualdad y se hace un énfasis particular en uno de los grupos laborales que más sufre de la condición de informalidad, el trabajo doméstico.

\section{Referencias}

Alaimo, V., Bosch, M., Kaplan, D., Pagés, C. y Ripani, L. (2015). Empleos para crecer. Banco Interamericano de Desarrollo.

Barbosa, E., Pereira, S. y Brandão, E. (2013). The Shadow Economy in Portugal: An Analysis Using the MIMIC Model. FEP Working Papers, 514.

Bosch, M. y Maloney, W. (2005). Labour Market Dynamism in Developing Countries: Comparative Analyses Using Continuous Time Markov Processes. World Bank Policy Research, Working Paper, 3583.

Castells, M. y A. Portes (1989). "World underneath: The origins, dynamics and effects of the informal economy." En The Informal Economy: Studies in Advanced and Less Developed Countries, editado por Portes et al. The Johns Hopkins University Press. 
Cebollada, M. (2016). Estudio comparativo de la legislación para el trabajo del hogar en América Latina. Consejo Nacional para Prevenir la Discriminación. Ciudad de México, México.

Dougherty, S. y Escobar, O. (2013). "The determinants of informality in Mexico's states". OECD.

Falco, P., Kerr, A., Rankin, N., Sandefur, J. y F. Teal (2011), "The returns to formality and informality in Urban Africa”, Labour Economics, Elsevier vol. 18(S1), páginas 523531.

Friedman, E. Johnson, S. Kaufmann, D. y Zoido-Lobaton, P. (2000). "Dodging the Grabbing hand: The Determinants of Unofficial Activity in 69 Countries". Journal of Publics Economics, 76, 459-493.

Frölich, M., Kaplan, D., Pagés, C., Rigolini J. y Robalino, D., Social Insurance, Informality, and Labor Markets: How to Protect Workers While Creating Good Jobs. Ed. Oxford.

Galli, R. y Kucera, D. (2003). Informal employment in Latin America: Movements over business cycles and the effects of worker rights. International Institute for Labour Studies Geneva.

Gërxhani, K. (2017). "The Informal Sector in Developed and Less Developed Countries: A Literature Survey". Public Choice, Vol. 120, No. 3/4, pp. 267-300.

Goldber, P. y Pavcnik, N. (2003). "The response of the informal sector to trade liberalization". National Bureau of Economic Research, working Paper 9443, Cambridge.

Gómez, J. (2012). Informalidad y tributación en América Latina: Explorando los nexos para mejorar la equidad. Foro LAC sobre Política Tributaria "Incentivos tributarios e informalidad en tiempos de consolidación fiscal”.

La Porta, R . y A. Shleifer (2014), "Informality and Development", Journal of Economic Perspectives, vol. 28, núm. 3, pp. 109-126.

(2008), “The Unofficial Economy and Economic Development”, Brookings Papers on Economic Activity, Economic Studies Program, vol. 39, núm. 2, (otoño), The Brookings Institution, pp. 275-363.

Levy, S. y Székely, M. (2016). "¿Más escolaridad, menos informalidad? Un análisis de cohortes para México y América Latina" .EL trimestre económico, vol. LXXXIII (4), núm. 332, octubre-diciembre, pp. 499-548.

Loayza, N.V. y Rigolini, L. (2006). Informality Trends and Cycle. World Bank Policy Research Working Papers, 4078.

Loayza, N., y Sugawara, N. (2009). "El sector informal en México: Hechos y explicaciones fundamentales". El Trimestre Económico, Vol. 76, No. 304, FCE, Octubre-diciembre, pp. 887-920. 
Martínez, S. J., Ruiz, C., Ruesga, S., Rosales, M., Cazes, S., y Falco, P., (2017), Informalidad: implicaciones legislativas y políticas públicas para reducirla. Instituto Belisario Domínguez, Senado de la República. México.

Organización Internacional del Trabajo (OIT) (2016). Protección social del trabajo doméstico: tendencias y estadísticas. Oficina Internacional del Trabajo, Departamento de Proteccio $\square$ n Social (SOCPRO) en colaboracio $\square$ n con INWORK, Ginebra, No. 16.

(2015). Formalizando la informalidad juvenil: experiencias innovadoras en México. Oficina Regional para América Latina y el Caribe, Lima: OIT.

(2014). Panorama Laboral Temático: Transición a la Formalidad en América Latina y el Caribe. Lima: OIT, Oficina Regional para América Latina y el Caribe.

(2013). Panorama Laboral 2013 para América Latina y el Caribe. OIT, Oficina Regional para América Latina y el Caribe, Lima.

(2012). Effective protection for domestic workers: a law to designing labour laws. International Labour Office, Conditions of Work and Employment Programme, Industrial and Employment Relations Department, Geneva.

(2011). Panorama Laboral 2011 para América Latina y el Caribe. OIT, Oficina Regional para América Latina y el Caribe, Lima.

en desarrollo.

(2009). La globalización y el empleo en el sector informal en los países

Organización para la Cooperación y el Desarrollo Económicos (OCDE) (2009). "Is Informal Normal? Towards more and better jobs in developing countries" Development Centre Studies, OECD Publishing.

Perry, G., Maloney, W., Arias, O., Fajnzylber, P., Mason, A. y Saavedra-Chanduvi, J. (2007). Informalidad: escape e inclusión. Banco Mundial, Washington, D.C.

Pollack, M. y C. Jusidman (1997). "El sector informal urbano desde la perspectiva de género: el caso de México.” CEPAL, Naciones Unidas.

Portes, A., M. Castells y L. Benton (1989). "The informal economy: Studies in Advanced and Less Developed Countries.” The Johns Hopkins University Press.

Portes, A. y Haller W. (2004). La economía informal. División de Desarrollo Social, Naciones Unidas, Santiago de Chile.

Ros, J. (2015). (2013), Algunas tesis equivocadas sobre el estancamiento económico de México, El Colegio de México, México.

Ruesga, S. M., Carbajo, D. y Pérez, M. (2013). "La economía sumergida y el ciclo económico". Atlantic Review of Economic, 2(2013), 1-37. 
Samaniego, N. (2008). "El crecimiento explosivo de la economía informal". Economíaunam, vol. 5, núm. 13, pp. 30-41. Universidad Nacional Autónoma de México.

Schneider, F. (2015). "Tax Losses due to Shadow Economy Activities in OECD Countries from 2011 to 2013: A preliminary calculation". IZA, Department of Economics, Johannes Kepler University of Linz.

Tokman, V. (1987). "El sector informal: Quince años después.” El Trimestre Económico No. 215. F. C. E.

Tokman, V. (2011). De la informalidad a la modernidad. Oficina Internacional del Trabajo OIT, 2001.

Willian, C.C. y Youseff, Y. (2014). "Combating Informal Employment in Latin America: A Critical Evaluation of the Neo-Liberal Policy Approach". Research in World Economy, 5(2). Recuperado de www.sciedu.ca/rwe.

\section{Autoempleo.}

En la literatura especializada se incluye un conjunto de estudios en los que se aborda el tema del autoempleo. En primera instancia se explora teóricamente la tensión que existe entre el autoempleo como emprendimiento y el autoempleo como estrategia de supervivencia. Por otra parte se hacen trabajos empíricos en los que se analizan los países y grupos poblacionales en los que se concentra en mayor proporción el fenómeno y se encuentran características particulares del autoempleo en los países en desarrollo y en los grupos de edad de jóvenes y personas mayores.

Se estudian las determinantes del carácter emprendedor en distintos sectores de la economía y se incluyen algunos textos sobre micro empresas y sobre micro financiamiento, enfoques que son fundamentales en el tema que ocupa si se considera que gran parte de la actividad económica informal se concentra en las micro y pequeñas empresas.

\section{Referencias}

Amarante, Veronica y Perazzo Ivone (2013), Trabajo por Cuenta Propia y Monotributo en Uruguay, Instituto de Economia, Uruguay.

Blanchflower, D. (2004). "Self-employment: More may not be better". Working Paper 10286, february, JEL No. J4

(1998). "Self-employment in OECD countries" National bureau of Economic Research, Working Paper. 
Blau, D. (1985). "Self-employment and self-selection in developing country labour markets." Southern Economic Journal, No. 52

Cueto, B. (2007). El empleo autónomo como forma de entrada al mercado de trabajo entre los jóvenes no universitarios. Departamento de Economía Aplicada.

Cunningham, W. y W. F. Maloney, (1998). "Heterogeneity among Mexico's microenterprises.” Policy Research Working Paper 1999, The World Bank.

Falco, P., Haywood, L. (2013). Entrepreneurship versus Joblessness: Explaining the Rise in Self-Employment. German Institute for Economic Research.

Farazi, S. (2014), Informal firms and financial inclusion. Status and determinants. Policy research working paper. No. 6778. The World Bank.

Fields, G. (2014). "Self-employment and poverty in developing countries" Iza World of Labor, Cornell University.

Di Giannatale, S., Ramírez-Abarca, G. y Smith, R. (2013). "Estimating the Effects of Formality on Mexican Informal Microfirms. A Joint Multivariate Approach". Economía Mexicana. Nueva Época, vol. II, 2013, pp. 441-463. Centro de Investigación y Docencia Económicas, A.C.

Kaplan, D. (2016). Formalización e inscripción al IMSS de los trabajadores independientes. División de Mercados Laborales y Seguridad Social, BID. Revista Animal Político.

Maloney, W. F. (2003). "Informal self-employment: Poverty trap or decent alternative," en Pathways Out of Poverty, de Fields, G. y G. Pfeffermann, eds., Boston, Kluwer.

(1999). "Self-employment and labour turnover. Cross-country evidence" Policy Research Working Paper 2102, The World Bank.

Pagán , J. y S. Sánchez (2001). "Gender issues in workforce participation and selfemployment in rural Mexico", en The Economics of Gender in Mexico: work, family, state and market. Correia, M. y E. Katz, eds. The World Bank. Washington, D.C.

Pietrobelli, C., Rabellotti, R. y Aquilina, M. (2004). "An empirical study of the Determinants of self-employment in developing countries." Journal of International Development, 16.

Schott, T., Rogoff, E., Herrington M. y Penny, K. (2017). GEM Special Report on Senior Entrepreneurship 2017. Global Entrepreneurship Research Association.

Temkin, B. (2009). "Informal Self-Employment in Developing Countries: Entrepreneurship or Survivalist Strategy? Some Implications for Public Policy". Analyses of Social Issues and Public Policy, Vol. 9, No. 1, pp. 135-156.

Van der Sluis, J., M. Van Praag y W. Vijverberg (2005). "Entrepreneurship selection and performance: A meta-analysis of the impact of education in developing countries". The World Bank Economic Review, Vol. 19, No. 2. 
Yamada, G. (1996). "Urban informal employment and self-employment in developing countries: Theory and evidence." Economic Development and Cultural Change, No. 44(2). 